\title{
Dietary fat and early-onset prostate cancer risk
}

\author{
Artitaya Lophatananon ${ }^{1,2}$, Jane Archer ${ }^{1}$, Douglas Easton ${ }^{3}$, Richard Pocock ${ }^{4}$, David Dearnaley ${ }^{5}$,
} Michelle Guy ${ }^{5}$, Zsofia Kote-Jarai ${ }^{5}$, Lynne O'Brien ${ }^{5}$, Rosemary A. Wilkinson ${ }^{5}$, Amanda L. Hall ${ }^{6}$, Emma Sawyer ${ }^{5}$, Elizabeth Page ${ }^{5}$, Jo-Fen Liu ${ }^{1}$, Sandra Barratt ${ }^{1}$, Aneela A. Rahman ${ }^{1}$, The UK Genetic Prostate Cancer Study Collaborators $\dagger$, British Association of Urological Surgeons' Section of Oncology $\dagger$, Rosalind Eeles ${ }^{4,6} \ddagger$ and Kenneth Muir ${ }^{1} \ddagger$

${ }^{1}$ Division of Epidemiology, University of Nottingham Medical School, Queen's Medical Centre, Nottingham NG7 2UH, UK

${ }^{2}$ Ministry of Public Health, Nonthaburi 11000, Thailand

${ }^{3}$ CR-UK Genetic Epidemiology Unit, Strangeways Research Laboratories, Worts Causeway, Cambridge CB1 8RN, UK

${ }^{4}$ Royal Devon and Exeter NHS Foundation Trust, Barrack Road, Exeter Ex2 5DW, UK

${ }^{5}$ The Institute of Cancer Research, 15 Cotswold Road, Sutton, Surrey, SM2 5NG, UK

${ }^{6}$ The Royal Marsden NHS Foundation Trust, Downs Road, Sutton SM2 5PT, UK

(Received 20 July 2009 - Revised 3 November 2009 - Accepted 5 November 2009 - First published online 19 January 2010)

The UK incidence of prostate cancer has been increasing in men aged $<60$ years. Migrant studies and global and secular variation in incidence suggest that modifiable factors, including a high-fat diet, may contribute to prostate cancer risk. The aim of the present study was to investigate the role of dietary fat intake and its derivatives on early-onset prostate cancer risk. During 1999-2004, a population-based case-control study with 512 cases and 838 controls was conducted. Cases were diagnosed with prostate cancer when $\leq 60$ years. Controls were sourced from UK GP practice registers. A self-administered FFQ collected data on typical past diet. A nutritional database was used to calculate daily fat intake. A positive, statistically significant risk estimate for the highest $v$. lowest quintile of intake of total fat, SFA, MUFA and PUFA was observed when adjusted for confounding variables: OR 2.53 (95\% CI 1.72, 3.74), OR 2.49 (95\% CI 1.69, 3.66), OR 2.69 (95\% CI $1.82,3.96$ ) and OR $2.34(95 \%$ CI $1.59,3.46)$, respectively, with all $P$ for trend $<0.001$. In conclusion, there was a positive statistically significant association between prostate cancer risk and energy-adjusted intake of total fat and fat subtypes. These results potentially identify a modifiable risk factor for early-onset prostate cancer.

Early-onset prostate cancer: Total fat: Fat subtypes: Case-control studies

Prostate cancer is a substantial cause of morbidity and mortality in westernised countries including the UK. In 2005, more than 34000 men were diagnosed with prostate cancer $^{(1)}$. Early-onset prostate cancer (age of diagnosis under 60) accounts for about $10 \%$ of the cases, but these subjects are more likely to have positive family history and are associated with a more aggressive disease course. Incidence in these younger men has increased between 1975 and 2005, yet the aetiology remains unclear. The gene-environment interactions in Prostate Cancer study were set up to investigate the role of genetics, as well as modifiable risk factors including high-fat diet, in the development of early-onset prostate cancer and their potential interactions.

Fat intake has been the focus of dietary studies and prostate cancer risk more than any other dietary components. Fat is the most energy-dense dietary constituent. It yields approximately $37.65 \mathrm{~J} / \mathrm{g}$ ( 9 calories $/ \mathrm{g})$ on complete oxidation. Chemically, fat is composed of a glycerine backbone with three fatty acids attached. The different fatty acids make one fat different from another. These fatty acids can be divided into three sub groups, SFA, MUFA and PUFA ( $n-6$ and $n-3$ fatty acids). Their biological properties vary by the degree of saturation and the length of the carbon chain ${ }^{(2)}$. It has been suggested that $40 \%$ of total energy intake in a traditional Western diet is derived from fat ${ }^{(3)}$. The relationship between dietary fat intake and prostate cancer has been proposed through the high correlation between per capita fat consumption and the large international difference in prostate cancer $\operatorname{rates}^{(4,5)}$. The early epidemiological evidence on fat and prostate cancer was consistent and suggested a possible causal association ${ }^{(6)}$. These studies, however, did not necessarily assess an independent effect of dietary fat and subsequent prostate cancer risk. Since fat is highly correlated with energy intake, energy adjustment is therefore recommended. Later

*Corresponding author: Dr Artitaya Lophatananon, fax +44 1212469 127, email alopha2003@yahoo.co.uk

$\dagger$ Lists available on request

$\ddagger$ Joint senior authors 
studies allow for energy adjustment and the findings across different types of epidemiological study become mixed. Cohort studies identified weak, positive, non-significant or null associations between prostate cancer risk and total fat intake. Evidence from case-control studies is more consistent with the majority observing positive associations particularly in advanced cases (OR for the highest compared with the lowest category of total fat intake varied from 1.08 to $3 \cdot 64)^{(3,7-18)}$. Very few studies, however, investigated dietary fat and early onset of prostate cancer. A study of a relatively young cohort was among those that observed a weak, positive and non-significant association (mean age at prostate cancer diagnosis of 60 years, range $47-67$ years $)^{(19)}$. Null or weak positive non-significant associations were observed for the five analyses of cases aged $<70$ years ${ }^{(13,18,20-22)}$.

In the present study, we investigate the independent effect of dietary fat intake in relation to early-onset prostate cancer. Dietary fat presented here refers to total fat, saturated and unsaturated fat (both MUFA and PUFA).

\section{Materials and methods}

\section{Study participants and data collection}

The gene-environment interactions in Prostate Cancer study is a multicentre, population-based case-control study conducted during 1999-2004. The present study was conducted according to the guidelines laid down in the Declaration of Helsinki, and all procedures involving human subjects/patients were approved by the Trent Multi-Centre Research Ethics Committee (MREC/99/4/013(Mar)). Written informed consent was obtained from all participants. Cases were men clinically diagnosed with prostate cancer at age less than or equal to 60 years. Cases were recruited through two approaches; firstly, consultants notified cases to the UK Genetic Prostate Cancer Study and secondly, the British Association of Urological Surgeons (BAUS) Section of Oncology database. Controls were randomly selected from the population list at GP practices where each case was registered. Controls were selected and matched by age to the case $( \pm 5$ years). The study identified 512 cases and 838 population frequency age-matched controls.

A self-administered postal questionnaire was used to assess exposure history including occupational history (for social class coding), smoking habit, physical activity, body shape and hair pattern, self-reported first degree family history of prostate cancer and diet. Diet was assessed using an adaptation of the validated semi-quantitative FFQ, developed by the MRC Dunn Nutritional Unit at Cambridge for the UK European Prospective Investigation of Cancer in Nutrition ${ }^{(21,23)}$. Study subjects were asked to recall what their usual diet was 5 years before prostate cancer diagnosis in the case group or to receiving the questionnaire in the control group. Participants were presented with a list of 130 food items, including foods that are main sources of nutrients in the average British $\operatorname{diet}^{(21)}$. They were asked to tick the box indicating how often each food was eaten based on a medium portion size; seven pre-defined frequencies range from 'never or less than once a month' to 'more than once a day'. Daily intake was calculated using the nutritional software to compute each nutrient from a total of 130 foods listed in the questionnaire and vitamin and mineral supplements. Nutrient composition values were from the Ministry of Agriculture, Fisheries and Food composition tables ${ }^{(24)}$.

All statistical analyses were undertaken using SPSS for Windows Version 14. An unconditional logistic regression was carried out to obtain OR and $95 \%$ CI. Energy adjustment was done using the residual method proposed by Willett \& Stampfer ${ }^{(25)}$. Each exposure was categorised into quintiles based on the distribution of energy-adjusted value in controls. Age, one or more first-degree relatives with prostate cancer, and baldness in the fourth decade of life were further adjusted in multivariate models. The prostate cancer family history was adjusted for due to potential genetic predisposition in cases. Baldness was adjusted for based on a statistically significant baseline difference between cases and controls and made a difference to the goodness of fit of the model (see Table 1). To assess the existence of dose-response relationships, tests for linear trend (two-tailed) across increasing quintiles of nutrient intake were performed, modelling categorical nutrient intakes as continuous variables in the multivariate model.

Finally, etiologic fractions, related to total fat and fat subtypes, were calculated using the Miettinen formula for multi-category indicator: $\mathrm{EF}=\Sigma_{i} \mathrm{CF}_{i} \times\left(\mathrm{SMR}_{i}-1\right) / \mathrm{SMR}_{i}$, where $\mathrm{EF}$ is the etiologic fractions, $\mathrm{CF}$ is the proportion of cases with the marker of increased risk and SMR is the standardised morbidity ratio in that subcategory ${ }^{(26)}$.

\section{Results}

Table 1 shows the characteristics of study subjects. The mean age of cases and controls was 55.7 years. There was a strong, highly significant increased risk of prostate cancer in those with at least one first-degree relative (OR 6.09, $95 \%$ CI $3.99,9.27, P<0.001)$. The distribution of cases and controls according to social class was very similar, reflected in there being no significant association between prostate cancer risk and social class. There was no significant association between prostate cancer risk and cigarette smoking. Severe baldness in the fourth decade of life, compared with no/negligible baldness, was associated with a weak, statistically significant increase in risk (OR 1.47, $95 \%$ CI 1.09, 1.98, $P=0.01$ ). Participants' perceived body shape and moderate physical activity at different decades in life were similar for cases and controls and were not associated with risk.

Results from Table 2 showed that the difference in mean average energy intake between cases and controls was highly significant $(P<0.001)$; mean average daily energy intake in cases was $12060.42 \mathrm{~kJ} / \mathrm{d}(2882.51 \mathrm{kcal} / \mathrm{d}), 9.8 \%$ higher than that of controls.

Overall, the diet of study subjects contained fat as approximately $30 \%$ of total energy intake. There was no significant difference in mean total fat intake between cases and controls when measured as a percentage of total energy $(P<0 \cdot 001)$. The actual total fat intake in cases though was $13 \%$ higher than that of controls. Similar percentage differences were also observed with fat subtypes.

Estimated risks are presented in Table 3. Moderate to strong, statistically significant OR were found for the highest three quintiles $v$. lowest quintile of total fat intake when adjusted only for age and for age, family history and baldness. For fat subtypes, moderate to strong, statistically significant 
Table 1. Characteristics of study participants

(OR values and $95 \% \mathrm{Cl}$ )

\begin{tabular}{|c|c|c|c|c|}
\hline Characteristics & Cases $(n 512)$ & Controls $(n 838)$ & OR & $95 \% \mathrm{Cl}$ \\
\hline Mean age at diagnosis (years) & $55 \cdot 7$ & $55 \cdot 7$ & 0.99 & $0.97,1.03$ \\
\hline \multicolumn{5}{|l|}{ Prostate cancer family history } \\
\hline At least one first-degree relative & $97(18.9)$ & $31(3.7)$ & 6.09 & $3.99,9.27$ \\
\hline \multicolumn{5}{|l|}{ Social class* } \\
\hline I, II & $239(53 \cdot 1)$ & $420(50 \cdot 1)$ & 1 & \\
\hline IIIN, IIIM & $185(39.4)$ & $303(38.3)$ & 1.07 & $0.84,1.37$ \\
\hline IV, V & $46(9 \cdot 8)$ & $68(8.6)$ & $1 \cdot 19$ & $0.79,1.79$ \\
\hline \multicolumn{5}{|l|}{ Baldness in $40 \mathrm{~s}^{*}$} \\
\hline None/negligible & $231(47 \cdot 3)$ & $415(51 \cdot 4)$ & 1 & \\
\hline Moderate & $150(30.7)$ & $261(32.3)$ & 1.03 & $0.80,1.34$ \\
\hline Severe & $107(21.9)$ & $131(16 \cdot 2)$ & 1.47 & $1.09,1.98$ \\
\hline \multicolumn{5}{|l|}{ Cigarette smoking } \\
\hline Never & $152(30.3)$ & $285(34.3)$ & 1 & \\
\hline Previous & $255(50 \cdot 8)$ & $394(47.4)$ & $1 \cdot 21$ & $0.94,1.56$ \\
\hline Current & $95(18.6)$ & $152(18.3)$ & 1.17 & $0.85,1.62$ \\
\hline \multicolumn{5}{|c|}{ Participants' perceived body shape full/obese $v$. thin/moderate reported at age } \\
\hline $20 \mathrm{~s}$ & $75(15 \cdot 2)$ & $100(12 \cdot 2)$ & 1.29 & $0.94,1.79$ \\
\hline $30 \mathrm{~s}$ & $171(34 \cdot 8)$ & $249(30.4)$ & 1.22 & $0.96,1.55$ \\
\hline $40 \mathrm{~s}$ & $337(69 \cdot 1)$ & $539(65.9)$ & $1 \cdot 16$ & $0.91,1.47$ \\
\hline \multicolumn{5}{|c|}{ Moderate physical activity reported at age } \\
\hline $20 \mathrm{~s}$ & $480(96 \cdot 2)$ & $790(96 \cdot 1)$ & 0.94 & $0.57,1.83$ \\
\hline $30 \mathrm{~s}$ & $476(95.4)$ & $784(95.4)$ & 1.00 & $0.59,1.70$ \\
\hline $40 \mathrm{~s}$ & $462(92 \cdot 8)$ & $758(92.4)$ & 0.84 & $0.69,1.61$ \\
\hline Last 5 years & $422(84.9)$ & $695(85.4)$ & 0.96 & $0.70,1.32$ \\
\hline
\end{tabular}

* Participants were categorised by social class by identifying the longest job held: I, professional; II, managerial and technical occupation; IIIN, non-manual skilled occupation; IIIM, manual skilled occupation; IV, partly skilled occupation; V, unskilled occupation.

OR were found for the highest three quintiles of intake $v$. the lowest when adjusted for age only, when further adjusted for family history and baldness, only the highest two quintiles $v$. lowest quintile remained significant. OR from the fully adjusted model ranged from 1.57 to 2.69 .

A highly significant trend was observed for increased risk of prostate cancer with increased intake of total fat and its subtypes across quintiles in both models.

\section{Etiologic fractions}

Assuming the relationship between fat intake and prostate cancer is causal; etiologic fraction results suggested that on average, about $42 \%$ of prostate cancer cases in the present study could be attributed to increased fat intake (greater than $64.48 \mathrm{~g} / \mathrm{d}$ ).

\section{Discussion}

We have conducted a UK nationwide prostate cancer casecontrol study with 512 early-onset cases and 838 population based controls. A self-administered FFQ was employed to collect typical dietary data. A value based on daily intake was assessed and the estimated risk was calculated based on quintile distribution. The results appear to support the hypothesis that increased total fat, SFA, MUFA and PUFA intakes increase the risk of prostate cancer diagnosed at a young age of 60 years or less. A positive, strong, statistically significant association between total fat and fat subtypes was observed (adjusted OR of the highest compared to the lowest quintile of intake were between 2.34 and 2.69).

Like other diet and cancer case-control studies, the present study could be subject to recall bias particularly in the control group. To evaluate the representativeness of the control group's nutrient intake, comparisons of crude mean intake

Table 2. Daily energy and total fat intake among 512 prostate cancer cases and 838 controls: UK 1999-2004

(Mean values and standard deviations with their mean difference)

\begin{tabular}{|c|c|c|c|c|c|c|c|}
\hline \multirow[b]{3}{*}{ Variable } & \multicolumn{4}{|c|}{ Crude nutrient intake } & \multirow[b]{3}{*}{ Mean difference } & \multirow[b]{3}{*}{$\%$} & \multirow[b]{3}{*}{$P$} \\
\hline & \multicolumn{2}{|c|}{ Cases } & \multicolumn{2}{|c|}{ Controls } & & & \\
\hline & Mean & SD & Mean & SD & & & \\
\hline Energy $(\mathrm{kJ} / \mathrm{d})$ & $12060 \cdot 42$ & $3572 \cdot 30$ & $10878 \cdot 60$ & $3260 \cdot 88$ & $1181 \cdot 81$ & $9 \cdot 8$ & $<0.001$ \\
\hline Total fat (\% energy) & 30.94 & 6.90 & 30.07 & 6.75 & 0.87 & $2 \cdot 8$ & 0.23 \\
\hline Total fat $(\mathrm{g} / \mathrm{d})$ & 99.40 & 38.65 & 86.45 & 32.73 & $12 \cdot 9$ & $13 \cdot 0$ & $<0.001$ \\
\hline Saturated fat $(\mathrm{g} / \mathrm{d})$ & $36 \cdot 13$ & $15 \cdot 37$ & $31 \cdot 22$ & $13 \cdot 13$ & 4.91 & $13 \cdot 6$ & $<0.001$ \\
\hline Polyunsaturated fat (g/d) & $18 \cdot 40$ & 8.89 & 16.46 & 8.03 & 1.94 & $10 \cdot 5$ & $<0.001$ \\
\hline Monounsaturated fat $(\mathrm{g} / \mathrm{d})$ & 33.91 & 13.33 & $29 \cdot 40$ & 11.41 & 4.51 & 13.3 & $<0.001$ \\
\hline
\end{tabular}


Table 3. Risk estimates according to quintile of fat intake and its derivatives; energy-adjusted residual method (OR values and $95 \% \mathrm{Cl}$ )

\begin{tabular}{|c|c|c|c|c|c|c|c|c|}
\hline \multirow[b]{3}{*}{ Nutrient } & & & & & \multicolumn{4}{|c|}{ Energy-adjusted nutrient intake } \\
\hline & \multicolumn{2}{|c|}{$\begin{array}{l}\text { Cases } \\
(n 512)\end{array}$} & \multicolumn{2}{|c|}{$\begin{array}{l}\text { Controls } \\
(n \text { 838) }\end{array}$} & \multicolumn{2}{|c|}{ Model $1^{*}$} & \multicolumn{2}{|c|}{ Model 2† } \\
\hline & $n$ & $\%$ & $n$ & $\%$ & OR & $95 \% \mathrm{Cl}$ & OR & $95 \% \mathrm{Cl}$ \\
\hline \multicolumn{9}{|l|}{ Total fat $(\mathrm{g} / \mathrm{d})$} \\
\hline Q1: $(<64 \cdot 48)$ & 59 & 11.5 & 167 & $19 \cdot 9$ & 1 & & 1 & \\
\hline Q2: (64.48-78.72) & 75 & $14 \cdot 6$ & 168 & $20 \cdot 0$ & $1 \cdot 26$ & $0.85,1.89$ & 1.21 & $0.79,1.85$ \\
\hline Q3: $(78 \cdot 72-91.66)$ & 107 & $20 \cdot 9$ & 168 & $20 \cdot 0$ & 1.80 & $1.22,2.64$ & 1.62 & $1.08,2.44$ \\
\hline Q4: (91.66-108.35) & 109 & $21 \cdot 3$ & 168 & $20 \cdot 0$ & 1.84 & $1.25,2.69$ & 1.58 & $1.06,2.38$ \\
\hline $\begin{array}{l}\text { Q5: }(>108 \cdot 35) \\
P \text { for trend }\end{array}$ & 162 & $31 \cdot 6$ & 167 & $19 \cdot 9$ & $2 \cdot 75$ & $\begin{array}{l}1.90,3.97 \\
<0.001\end{array}$ & $2 \cdot 53$ & $\begin{array}{l}1.72,3 \cdot 74 \\
<0.001\end{array}$ \\
\hline \multicolumn{9}{|l|}{ Saturated fat $(g / d)$} \\
\hline Q1: $(<23.09)$ & 61 & 11.9 & 167 & $19 \cdot 9$ & 1 & & 1 & \\
\hline Q2: (23.09-28.43) & 78 & $15 \cdot 2$ & 168 & $20 \cdot 0$ & 1.26 & $0.85,1.88$ & 1.18 & $0.77,1.80$ \\
\hline Q3: (28.43-33.14) & 93 & $18 \cdot 2$ & 168 & $20 \cdot 0$ & 1.52 & $1.03,2.23$ & 1.37 & $0.91,2.07$ \\
\hline Q4: (33.14-39.29) & 114 & $22 \cdot 3$ & 168 & $20 \cdot 0$ & 1.86 & $1 \cdot 27,2 \cdot 71$ & 1.60 & $1.07,2.39$ \\
\hline $\begin{array}{l}\text { Q5: }(>39.29) \\
P \text { for trend }\end{array}$ & 166 & $32 \cdot 4$ & 167 & $19 \cdot 9$ & $2 \cdot 72$ & $\begin{array}{l}1.89,3.92 \\
<0.001\end{array}$ & 2.49 & $\begin{array}{l}1.69,3.66 \\
<0.001\end{array}$ \\
\hline \multicolumn{9}{|c|}{ Monounsaturated fat (g/d) } \\
\hline Q1: $(<22 \cdot 18)$ & 59 & 11.5 & 167 & $19 \cdot 9$ & 1 & & 1 & \\
\hline Q2: (22.18-26.73) & 78 & $15 \cdot 2$ & 168 & $20 \cdot 0$ & 1.31 & $0.88,1.96$ & 1.24 & $0.82,1.90$ \\
\hline Q3: (26.73-31.08) & 97 & 18.9 & 168 & $20 \cdot 0$ & 1.63 & $1 \cdot 10,2 \cdot 40$ & 1.48 & $0.98,2.23$ \\
\hline Q4: $(31.08-36.52)$ & 109 & $21 \cdot 3$ & 168 & $20 \cdot 0$ & 1.84 & $1.25,2.69$ & 1.57 & $1.05,2.36$ \\
\hline $\begin{array}{l}\text { Q5: }(>36.52) \\
P \text { for trend }\end{array}$ & 169 & 33.0 & 167 & $19 \cdot 9$ & $2 \cdot 87$ & $\begin{array}{l}1.99,4.14 \\
<0.001\end{array}$ & $2 \cdot 69$ & $\begin{array}{l}1.82,3.96 \\
<0.001\end{array}$ \\
\hline \multicolumn{9}{|l|}{ Polyunsaturated fat $(\mathrm{g} / \mathrm{d})$} \\
\hline Q1: $(<12 \cdot 10)$ & 60 & $11 \cdot 7$ & 167 & $19 \cdot 9$ & 1 & & 1 & \\
\hline Q2: $(12 \cdot 10-14 \cdot 68)$ & 75 & 14.6 & 168 & $20 \cdot 0$ & 1.24 & $0.83,1.86$ & 1.15 & $0.75,1.75$ \\
\hline Q3: (14.68-17.22) & 95 & $18 \cdot 6$ & 168 & $20 \cdot 0$ & 1.57 & $1.06,2.31$ & 1.40 & $0.93,2 \cdot 11$ \\
\hline Q4: $(17.22-20.49)$ & 125 & 24.4 & 168 & $20 \cdot 0$ & 2.07 & $1.42,3.01$ & 1.80 & $1.21,2.68$ \\
\hline $\begin{array}{l}\text { Q5: }(>20 \cdot 49) \\
P \text { for trend }\end{array}$ & 157 & $30 \cdot 7$ & 167 & $19 \cdot 9$ & $2 \cdot 62$ & $\begin{array}{l}1.81,3.78 \\
<0.001\end{array}$ & $2 \cdot 34$ & $\begin{array}{l}1.59,3.46 \\
<0.001\end{array}$ \\
\hline
\end{tabular}

$Q$, reference quintile.

Values in each quintile were mutually exclusive; cut points are given to two decimal places.

${ }^{*}$ Model 1 , adjusted for age as a continuous variable.

† Model 2, adjusted for age as a continuous variable, first-degree relative with prostate cancer and baldness in 40s.

of controls in the present study were made with The National Diet and Nutrition Survey by Henderson et al. ${ }^{(27)}$ and that of controls from a UK case-control study of diet and prostate cancer by Key et al. ${ }^{(11)}$.

The National Diet and Nutrition Survey was chosen as the sampling methods attempted to recruit a nationally representative sample and thorough methods were used to establish diet (both interviews and 7-d weighed records). Controls' mean daily intake of total and SFA was virtually the same as that reported in this survey (the survey reported daily intake values of 86.5 and $32.5 \mathrm{~g})^{(27)}$. However, controls' mean daily energy intake was higher with the survey-reported values of $9719.43 \mathrm{~kJ}(2323 \mathrm{kcal})$ in adult men $(19-64$ years $)$ as compared to $10878.40 \mathrm{~kJ}(2600 \mathrm{kcal})$ in our controls reflecting the lower percentage of energy from fat and fat subtypes in study controls. Department of Health suggested dietary reference values for energy for healthy men were $10669 \cdot 20 \mathrm{~kJ}(2550 \mathrm{kcal})^{(28)}$. Study controls had similar energy intake but consistently and substantially lower intakes of fat and fat derivatives than controls in the study by Key et al:; intake was $34 \%$ lower for total fat and MUFA and over $50 \%$ lower for SFA. Key et al. ${ }^{(11)}$ acknowledged that the average fat intake in their study was high. We therefore concluded that study controls could include some over-representation of health-conscious controls; however, any recall bias occurred was considered minimal.

In terms of case and control characteristics, it is important to highlight that all of these cases were diagnosed clinically in younger men presenting with significant disease (not through prostate specific antigen measurement/screening). As for the controls, there is a possibility of low-grade cases being included in the control group, as is the situation with all case-control studies using community-derived controls, but the potential size of this effect is less due to the younger age group on which the present study focuses. We therefore suggest that the above factors are unlikely to have had a significant effect on the results presented.

The positive association between total fat and prostate cancer risk observed is concordant with most case-control studies in the literature review where all stages of prostate cancer were combined. Of these, only three reported a statistically significant increased risk, also of moderate to strong magnitude ${ }^{(29-31)}$.

The only UK case-control study identified in the literature review did not specifically look at younger cases and found a non-significant inverse association between prostate cancer risk and high $v$. low total fat intake (OR 0.85, $95 \%$ CI 0.57 , 1.25); the lack of a significant association is thought to be attributable to participants' narrow range of high fat intake ${ }^{(32)}$. 
Increased saturated fat intake is positively associated with early-onset prostate cancer risk. The positive association observed is in keeping with approximately half of the casecontrol studies regardless of their age group identified in the literature review ${ }^{(7,3,29,31,32)}$.

Not all of the findings from previous studies supported a MUFA role in prostate cancer aetiology. This inconsistency may be partly related to the food sources of MUFA in different populations (e.g. meat in Western diet and olive oil in Mediterranean diet), as other components in the food sources may confound the effects of MUFA. One analysis of aggressive prostate cancer and another of early-onset prostate cancer observed statistically significant point estimates of lesser magnitude to those in the present study (OR 2.00, $95 \%$ CI $1.03,3.87)$ and (OR $1.9,95 \%$ CI $1 \cdot 1,3 \cdot 4)^{(13,20)}$.

The literature supporting an increased prostate cancer risk with high PUFA intake is inconsistent. One study reported a statistically significant $\mathrm{OR}$ with a significant $P$ for trend across quintiles ${ }^{(33)}$. Conversely, five studies suggested inverse $\operatorname{associations}^{(7,13,15,20,34)}$.

A high fat intake may increase the levels of endogenous androgens ${ }^{(34)}$. There are different mechanisms proposed depending on the type of fat. Polyunsaturated fat is prone to oxidation, generating lipid radicals and hydroperoxides that cause DNA damage leading to carcinogenesis ${ }^{(2)}$. The synthesis of PG and leukotrienes is regulated by fatty acid. PG and leukotrienes affect cell proliferation and metastases ${ }^{(3,35)}$. There may be an association between hypercholesterolaemia and prostate cancer ${ }^{(36,37)}$. Additionally, cholesterol is a major substrate for androgen synthesis. This may suggest that dietary fat influences prostate cancer risk though being a major determinant of plasma cholesterol levels. Other forms of fatty acid play a part in protecting against carcinogenesis. Fish oil, which rich in $n-3$ fatty acids EPA and DHA, may increase the accumulation of lipid peroxidation products in tumour tissue. These products subsequently decrease cell proliferation or increase cell death ${ }^{(3)}$.

The consistent results observed in the present study suggest that total fat intake is implicated in young-onset prostate cancer risk; thus, variation in risk estimates would be expected if a specific fat derivative was conferring risk, unless SFA, MUFA and PUFA were so highly collinear that they were not mutually exclusive. Also, early-onset prostate cancer cases are distinct from the case mix used in previous studies. A greater proportion of aggressive phenotypes would be expected to be more strongly associated with risk of prostate cancer due to fat intake. The greater risk seen in the present study could also be explained by the confounding interaction between the genetic component and the exposure. The younger-aged case series have a greater proportion due to genetic predisposition than older cases. The effects of the interaction could either be multiplicative or additive.

One might suggest that cases are overeating or overreporting the amount of food eaten. A post hoc analysis of protein and carbohydrate intake was conducted to investigate the possibility of overeating or over-reporting of all macronutrients by cases. Cases had approximately $10 \%$ higher mean daily intake of protein and carbohydrate, a difference which was highly statistically significant $(P<0 \cdot 001)$. This suggested that overeating or over-reporting by cases was plausible. On further multivariate analysis of protein and carbohydrate intakes, moderate to strong, positive, statistically significant $\mathrm{OR}$ in the highest $v$. lowest quintile of intake (when adjusted for age, family history and baldness) were observed. When adjusted for fat intake, the increased risk for protein and carbohydrate was no longer significant. This suggests that the risk conferred by protein and carbohydrate was due to confounding by fat intake and that while cases do eat more macronutrients than controls, it is the over-consumption of fat intake that is particularly associated with prostate cancer risk.

Given that the residual method of energy adjustment should differentiate the effects of food intake overall from those of diet composition ${ }^{(25)}$, the significant associations observed when adjusted for energy intake may not be caused by excess total nutrient intake or, by implication, excess energy intake of cases.

The positive, statistically significant associations observed indicate that 5 years before diagnosis could be the aetiologically relevant time for exposure to dietary fat, or whatever fat intake is a marker of, in relation to prostate cancer risk. Alternatively, it could show that intake 5 years before diagnosis is highly correlated with the true aetiologically relevant exposure period. Equally, subjects could report their typical diet, which has not changed during the last decade; thus, the findings could reflect the relevant time up to a decade before diagnosis. Considering this time implication together with the aggressive disease in our cases, high fat intake may play a role in both initiation and progression.

\section{Conclusion}

The present study observed an association between an earlyonset prostate cancer risk and a relatively high intake of total fat and fat derivatives. There was a strong, statistically significant positive risk estimate for high $v$. low intake of total fat, SFA, MUFA and PUFA; each risk estimate was of similar magnitude (OR between 2.34 and 2.69). A highly significant dose-response relationship was also observed for total fat and fat subtypes ( $P$ for trend $<0.001)$, suggesting an increase in the risk of prostate cancer with increase in fat intake. Etiologic fractions suggest that if these associations are causal, approximately $40 \%$ of early-onset prostate cancer cases could have an aetiology related to their fat intake. This has implications for prevention.

\section{Acknowledgements}

The study has no conflicts of interest. The present research was supported by the Prostate Cancer Research Foundation and Cancer Research UK grant C5047/A3354. The authors thank the UK Genetic Prostate Cancer Study Collaborators and the British Association of Urological Surgeons' Section of Oncology for their collaboration on the study. The original authors and their contributions are as follows: A. L. was involved in the conduct of the study, analysed the data and drafted the paper. J. A. was involved in the data quality control and analysed the data. D. E., R. P., D. D., M. G., Z. K.-J. L. O'B., R. A. W., A. L. H., E. S. and E. P. were involved in the development of the protocol, conduct of the study and revision of the paper. J.-F. L., S. B. and A. A. R. were involved in the data management, data collection and 
updated diet calculation programme. R. E. and K. M. were involved in the development of the protocol, conduct of the study and revision of the paper, and they are the principal investigator and guarantor.

\section{References}

1. Cancer Research UK (2008) UK prostate cancer incidence statistics. http://info.cancerresearchuk.org/cancerstats/types/prostate/ index.htm?script=true

2. Kolonel LN (2001) Fat, meat, and prostate cancer. Epidemiol Rev 23, 72-81.

3. Pandian SS, Eremin OE, McClinton S, et al. (1999) Fatty acids and prostate cancer: current status and future challenges. $J$ R Coll Surg Edinb 44, 352-361.

4. Gann PH (2002) Risk factors for prostate cancer. Rev Urol 4, Suppl. 5, S3-S10.

5. Kolonel LN (1996) Nutrition and prostate cancer. Cancer Causes Control 7, 83-94.

6. Kolonel LN, Nomura AMY \& Cooney RV (1999) Dietary fat and prostate cancer: current status. J Natl Cancer Inst 91, 414-428.

7. Andersson SO, Wolk A, Bergstrom R, et al. (1996) Energy, nutrient intake and prostate cancer risk: a population-based case-control study in Sweden. Int J Cancer 68, 716-722.

8. Chan JM, Pietinen P, Virtanen M, et al. (2000) Diet and prostate cancer risk in a cohort of smokers, with a specific focus on calcium and phosphorus (Finland). Cancer Causes Control 11, 859-867.

9. Deneo-Pellegrini H, Stefani ED, Ronco A, et al. (1999) Foods, nutrients and prostate cancer: a case-control study in Uruguay. Br J Cancer 80, 591-597.

10. Hayes RB, Ziegler RG, Gridley G, et al. (1999) Dietary factors and risks for prostate cancer among blacks and whites in the United States. Cancer Epidemiol Biomarkers Prev 8, 25-34.

11. Key TJ, Silcocks PB, Davey GK, et al. (1997) A case-control study of diet and prostate cancer. Br J Cancer 76, 678-687.

12. Kolonel LN, Yoshizawa CN \& Hankin JH (1988) Diet and prostatic cancer: a case-control study in Hawaii. Am J Epidemiol 127, 999-1012.

13. Kristal AR, Cohen JH, Qu P, et al. (2002) Associations of energy, fat, calcium, and vitamin D with prostate cancer risk. Cancer Epidemiol Biomarkers Prev 11, 719-725.

14. Ohno Y, Yoshida O, Oishi K, et al. (1988) Dietary beta-carotene and cancer of the prostate: a case-control study in Kyoto, Japan. Cancer Res 48, 1331-1336.

15. Ramon JM, Bou R, Romea S, et al. (2000) Dietary fat intake and prostate cancer risk: a case-control study in Spain. Cancer Causes Control 11, 679-685.

16. Rohan TE, Howe GR, Burch JD, et al. (1995) Dietary factors and risk of prostate cancer: a case-control study in Ontario, Canada. Cancer Causes Control 6, 145-154.

17. Turkes A (2000) Can diet reduce the risk of prostate cancer? In Challenges in Prostate Cancer, pp. 3-6 [W Bowsher, editor]. Malden, MA: Blackwell.

18. West DW, Slattery ML, Robison LM, et al. (1991) Adult dietary intake and prostate cancer risk in Utah: a case-control study with special emphasis on aggressive tumors. Cancer Causes Control 2, 85-94.

19. Veierod MB, Laake P \& Thelle DS (1997) Dietary fat intake and risk of prostate cancer: a prospective study of 25708 Norwegian men. Int J Cancer 73, 634-638.

20. Bidoli E, Talamini R, Bosetti C, et al. (2005) Macronutrients, fatty acids, cholesterol and prostate cancer risk. Ann Oncol 16, $152-157$.

21. Bingham SA, Gill C, Welch A, et al. (1997) Validation of dietary assessment methods in the UK arm of EPIC using weighed records, and 24-hour urinary nitrogen and potassium and serum vitamin $\mathrm{C}$ and carotenoids as biomarkers. Int $J$ Epidemiol 26, S137-S151.

22. Hodge AM, English DR, McCredie MR, et al. (2004) Foods, nutrients and prostate cancer. Cancer Causes Control 15, $11-20$.

23. Riboli E (1992) Nutrition and cancer: background and rationale of the European Prospective Investigation into Cancer and Nutrition (EPIC). Ann Oncol 3, 783-791.

24. Ministry of Agriculture Fisheries and Foods (1993) Annual Reports of National Food Survey Committee. London: HMSO.

25. Willett W \& Stampfer M (1998) Implications of total energy intake for epidemiologic analyses. In Nutritional Epidemiology, pp. 273-301 [W Willet, editor]. Oxford: Oxford University Press.

26. Miettinen OS (1974) Proportion of disease caused or prevented by a given exposure, trait or intervention. Am J Epidemiol 99, 325-332.

27. Henderson L, Gregory J \& Irving K (2003) The National Diet and Nutrition Survey. London: HMSO.

28. Department of Health (1991) Dietary Reference Values for Food Energy and Nutrients for the United Kingdom. London: HMSO.

29. Lee MM, Wang RT, Hsing AW, et al. (1998) Case-control study of diet and prostate cancer in China. Cancer Causes Control 9, 545-552.

30. Vlajinac HD, Marinkovic JM, Ilic MD, et al. (1997) Diet and prostate cancer: a case-control study. Eur J Cancer 33, 101-107.

31. Whittemore AS, Kolonel LN, Wu AH, et al. (1995) Prostate cancer in relation to diet, physical activity, and body size in blacks, whites, and Asians in the United States and Canada. J Natl Cancer Inst 87, 652-661.

32. Lagiou A, Trichopoulos D, Tzonou A, et al. (2001) Are there age-dependent effects of diet on prostate cancer risk? Soz Praventivmed 46, 329-334.

33. Tzonou A, Signorello LB, Lagiou P, et al. (1999) Diet and cancer of the prostate: a case-control study in Greece. Int $J$ Cancer 80, 704-708.

34. Hill P, Wynder EL, Garbaczewski L, et al. (1979) Diet and urinary steroids in black and white North American men and black South African men. Cancer Res 39, 5101-5105.

35. Ghosh J \& Myers CE Jr (1998) Arachidonic acid metabolism and cancer of the prostate. Nutrition 14, 48-49.

36. Bravi F, Scotti L, Bosetti C, et al. (2006) Self-reported history of hypercholesterolaemia and gallstones and the risk of prostate cancer. Ann Oncol 17, 1014-1017.

37. Freeman MR \& Solomon KR (2004) Cholesterol and prostate cancer. J Cell Biochem 91, 54-69. 